ESAIM: PROCEEDINGS, October 2007, Vol. 22, 118-121

Gabriel Caloz \& Monique Dauge, Editors

\title{
MICROMAGNETIC MODELING ON SELF-ASSEMBLED IRON NANOSTRUCTURES
}

\author{
Jean-Christophe Toussaint, Olivier Fruchart and Fabien Cheynis ${ }^{1}$
}

\begin{abstract}
Single-crystalline Fe dots self-assembled under ultra high vacuum (UHV) are used as a model system to discuss micromagnetic properties of sub-micron size magnetic dots. Landau and diamond states were identified by magnetic force microscopy (MFM) and reproduced by simulations.

To understand mechanisms involved in the magnetization reversal, the in-plane angular variations of nucleation and annihilation fields of a multi-domain magnetic single dot with a micro-SQUID were studied.
\end{abstract}

Résumé. Des îlots de fer auto-assemblés obtenus sous ultravide (UHV) sont utilisés comme système modèle pour étudier les propriétés micromagnétiques de plots sub-microniques. Les états Landau et losange ont été identifiés et reproduits par simulations. Pour comprendre les mécanismes de renversement de l'aimantation, les variations angulaires des champs de nucléation et d'annihilation d'un plot unique multi-domaines ont été étudiées par microsquid.

\section{INTRODUCTION}

The recent advances in micro fabrication techniques have stimulated interest in the study of properties of submicron sized magnetic elements. Promising applications including magnetic random access memory, highdensity recording media and magnetic sensors require a better understanding of their magnetic behavior on a mesoscopic scale. Coupled to experimental studies, micromagnetic simulations become a powerful tool to predict and to analyze the magnetic behavior of such small elements.

\section{REMANENT MAGNETIC CONFIGURATIONS}

The Fe(110) epitaxial dots were fabricated with pulsed laser deposition in ultra-high vacuum by self-assembly on $\mathrm{Mo}(110)[8 \mathrm{~nm}] / \mathrm{Al}_{2} \mathrm{O}_{3}(11 \overline{2} 0)$. The dots display the shape of ingots with atomically-flat facets, bulk lattice parameter and bulk cubic magneto-crystalline anisotropy $K_{1}$ favoring $<100>$ axes, however of magnitude much smaller than $\frac{1}{2} \mu_{0} M_{\mathrm{s}}^{2}[1]$. We focus on dots approximately $600 \times 300 \times 60 \mathrm{~nm}$ which display simple multi-domain states [2].

Hysteresis loops were performed at $300 \mathrm{~K}$ over assemblies of dots by Vibrating Sample Magnetometry. Magnetic force microscopy (MFM) was performed at $300 \mathrm{~K}$ using a Nanoscope IIIA (Multimode) from Digital Instruments with a lift height of about $25 \mathrm{~nm}$ and a Si tip coated with $40 \mathrm{~nm}$ of vertically magnetized CoCr. Micromagnetic simulations were performed at $0 \mathrm{~K}$ (no thermal activation) by integrating LLG equations using the finite differences code GL_FFT (CLab. Louis Néel) [3]. The sample was placed into a grid made of $128 \times 64 \times 16$ parallelepipedic cells with lateral and vertical size $h_{x}=h_{y}=4.6875 \mathrm{~nm}$ and $h_{z}=3.7500 \mathrm{~nm}$, respectively. We

\footnotetext{
1 Laboratoire Louis Néel, CNRS-INPG, 25, avenue des martyrs, BP 166, 38042 Grenoble Cedex, France
}

(c) EDP Sciences, SMAI 2007 
(a)

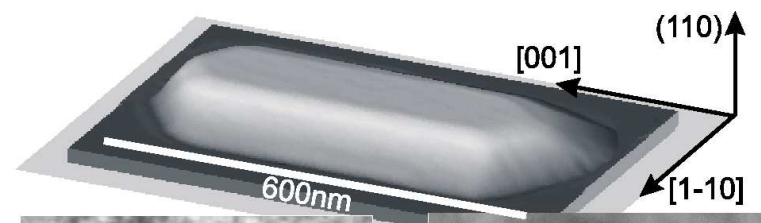

(b)

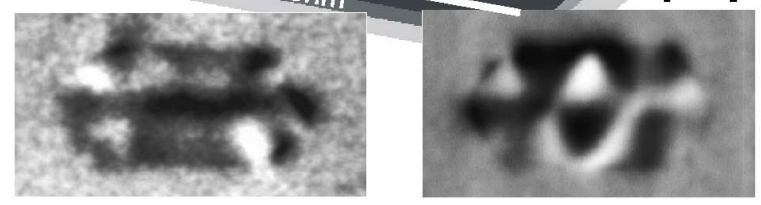

(c)
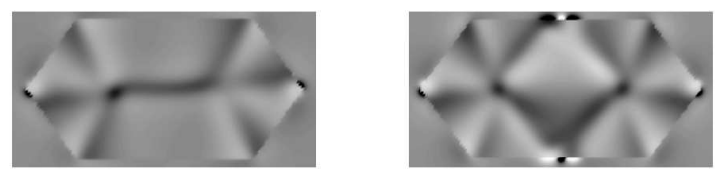

(d)

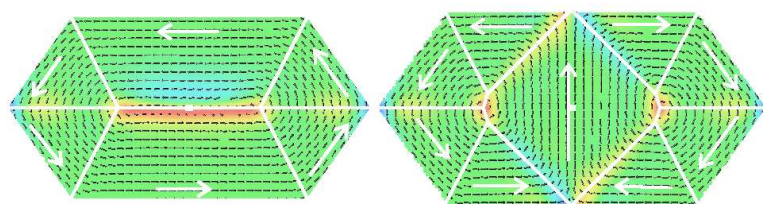

Figure 1. (a) $3 \mathrm{D}$ AFM image of a $600 \times 300 \times 60 \mathrm{~nm}$ Fe dot (true vertical scale). Below are shown (b) experimental MFM images after saturation along [001], (c) simulated $d H / d z$ maps over the dot with a lift height of $30 \mathrm{~nm}$, and (d) simulated configurations superimposed here with the Van den Berg construction, for both Landau (left) and diamond (right) states. Note the bipolar contrast of Néel walls and the unipolar contrast of the Bloch wall in (b-c). In (d) the color reveals the perpendicular component of magnetization of the mid-height plane, while white arrows sketch the in-plane magnetization direction.

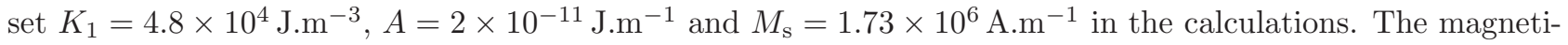
zation vector at each node is estimated as a second order interpolation of the vector field between the cell and its nearest neighbors, thus allowing the volume charges to vary linearly. The accuracy of our approach reaches the precision of the model B proposed by Ramstöck et al. [4]. In the reported calculations the dots edges were taken vertical to avoid discretizing artefacts on the side facets and simplify the comparison with flat dots. No significant difference was found for calculations performed with tilted facets. The magnetic anisotropy was that of bulk Fe and the external field was applied $0.1^{\circ}$ off high symmetry directions to avoid numerical artefacts.

Hysteresis loops of assemblies of dots were found to display zero remanence in any direction [1]. Mostly two types of remanent states were observed when imaging an assembly of dots at zero external field with MFM (Figure 1b). Simulated MFM contrast assuming a unipolar tip (Figure 1c) allows us to identify the so-called Landau state (LS, left) and diamond state (DS, right). These two states are well known for flat dots well above micron-size, and are explained by the Van den Berg (VdB) model [5]. Surprisingly the locus of magnetic walls and vortices in the simulated states is very close to the predictions of VdB down to tiny details like the slight vertical elongation of the two vortices in the DS (Figure 1d). Indeed the VdB model relies on three hypotheses, none of which is fulfilled in our case: infinitely soft magnetic material, infinitely thin plate, infinitely large sample (the latter being equivalent to zero exchange). Paradoxically other micron-size systems that fulfill all three criteria better than the present dots, like thin micron-size plates of Permalloy, were shown to deviate from VdB predictions [6]. We explain this apparent contradiction the following way. Firstly dipolar energy increases with the dot's height, so that both dipolar and anisotropy energies compress domain walls, reducing characteristic length scales (and removing domain wall tails) to a scale smaller than the dot's lateral size. Secondly, as in our case dipolar energy dominates over anisotropy energy, the locus of the walls and vortices is determined mainly by dipolar energy, and therefore coincides with $\mathrm{VdB}$ predictions.

To go further in our analysis of the magnetic structure of such systems, the internal structure of walls and vortices was examined by making cross-sections of both LS and DS. The top and bottom plane views of the LS show that look very different from the mid-height view (Figure 2a), due to the formation of Néel caps that reduce the amount of magnetic charges at both surfaces. The lateral cross section along the long direction $x$ (Figure $2 \mathrm{~b}$ ) reveals that the Bloch wall has the same topology as a single vortex: starting from a vortex, one can 'fabricate' a wall by driving apart the top and bottom ends and stretch laterally the mid-height core. A close examination of the middle plane view in (a) also shows that the wall is slightly bent downwards, although equilibrium is reached in the simulation. Figure $2 \mathrm{c}$ reveals also that the wall is an asymmetric Bloch wall, it helps reducing volume magnetic charges (arising from Néel caps) at the expense of exchange [7]. MFM resolution 

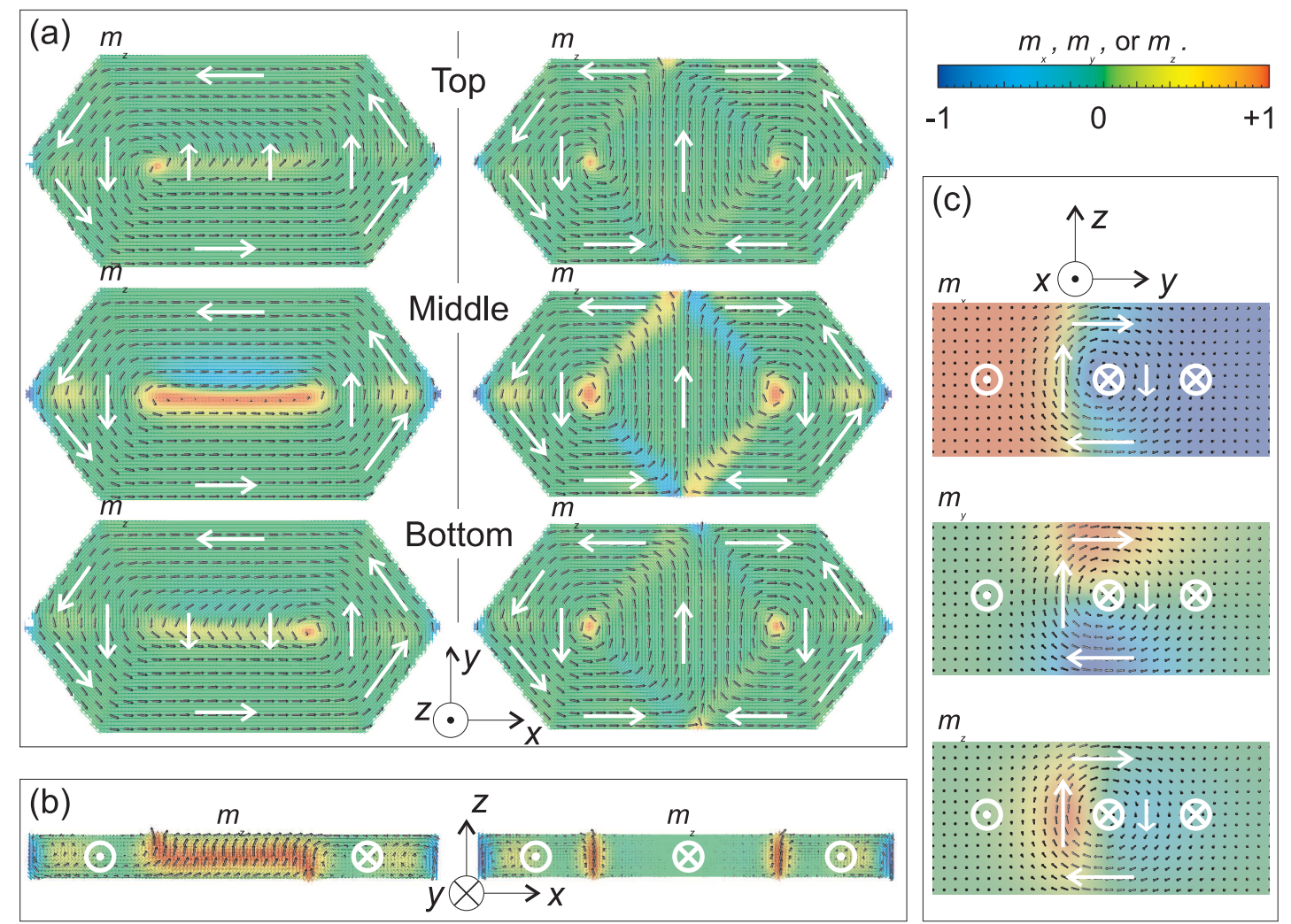

Figure 2. Simulated cross-sections of Landau (left in a-b) and diamond (right in a-b) states. $x=[001], y=[1 \overline{1} 0], z=(110)$. White arrows sketch the in-plane magnetization direction. The color code applies to $m_{z}$ in (a-b) and to successively all directions in (c). (a) top, middle and bottom plane views (b) middle cross-sections in the $x z$ plane (c) a partial middle cross-section of size $60 \times 130 \mathrm{~nm}$ in the $y z$ plane.

is not high enough to confirm that the experimental Bloch walls are asymmetric. The vortices in the DS are also influenced by the finite dot thickness: the core size is shrunk close to both surfaces to minimize surface magnetic charges. The surface and mid-height core half-width are $2 \Lambda_{\text {ex. }}$ and $3.4 \Lambda_{\text {ex. }}$, respectively, similar to the values predicted for $60 \mathrm{~nm}$ thickness using a variational model and a perfectly soft material: $2.2 \Lambda_{\text {ex }}$. and $5 \Lambda_{\text {ex. }}$, respectively [7], with $\Lambda_{\text {ex. }}=\sqrt{2 A / \mu_{0} M_{\mathrm{s}}^{2}}\left(\Lambda_{\text {ex. }}=3.3 \mathrm{~nm}\right.$ for Fe). Note also that the mid-height core seems to stretch along $y$ and reproduce the short segment of wall predicted by the VdB model (Figure 1d). If that piece of $\mathrm{VdB}$ segment was longer it is probable that each vortex would lower its symmetry and break into a short Bloch wall, like in the LS.

\section{Angular Dependence of MAGnetization SWITChing}

The angular dependence of the switching fields of a single dot was studied with the micro-SQUID technique [8]. This can be seen as the first experimental generalization of astroids for an individual multi-domain system. A suprising feature is the occurrence of discontinuities (named jumps hereafter) in $H_{\text {swi }}(\varphi)$ plots.

The in-plane $H_{\text {swi }}$ 's of a single Fe dot were measured below $4 \mathrm{~K}$.

The location and shape of the single dot under investigation were checked a posteriori by Atomic Force Microscopy (AFM). The size of the dot selected here (Figure 3a) is $420 \times 200 \times 30 \mathrm{~nm}$ (Figure 3b). 
The applied field was increased step-wise in hysteresis loops. The sample was divided into cells with uniform lateral and vertical size $h_{x}=h_{y}=4.70 \mathrm{~nm}$ and $h_{z}=$ $3.75 \mathrm{~nm}$, respectively.

In the following $\varphi$ denotes the angle between the in-plane $H_{\text {ext }}$ and the in-plane long axis of the dot [001] (Figure 3b). Due to a shape effect, in-plane [110] $\left(\varphi=90^{\circ}\right)$ is a magnetically-harder direction than [001]. The insets of Figure 3c show micro-SQUID hysteresis loops for two angles $\left(\varphi=+6 ;+90^{\circ}\right)$. Such loops with negligible remanence although with significant hysteresis, are characteristic of multidomain systems with a limited number of domains. Starting from positive saturation the first $H_{\text {swi }}$, named hereafter $H_{\text {nuc }}$, is expected to reveal a nucleation event, e.g. the entry of a magnetic vortex [9] in the dot. The second $H_{\text {swi }}$, occurring at negative fields and named $H_{\mathrm{ann}}$, is expected to reveal an annihilation event, i.e. the expulsion from the dot of a previously-nucleated vortex or wall. These mechanisms were confirmed by simulation. Figure 3c shows the experimental angular variation of $H_{\text {nuc }}(\varphi)$ and $H_{\text {ann }}(\varphi)$.

Two suprising features are observed. First, depending on the range of angles, one or two $H_{\text {nuc }}$ and/or $H_{\text {ann }}$ are observed. Second, jumps of both $H_{\text {nuc }}$ and $H_{\text {ann }}$ occur at some angles. These jumps were reproduced and understood with the help of numerical micromagnetism. They result from bifurcations, related to the interplay of the non-uniform magnetization with the shape of the dot. This also shows that a complex $H_{\text {swi }}$ behavior does not necessarily result from defects.
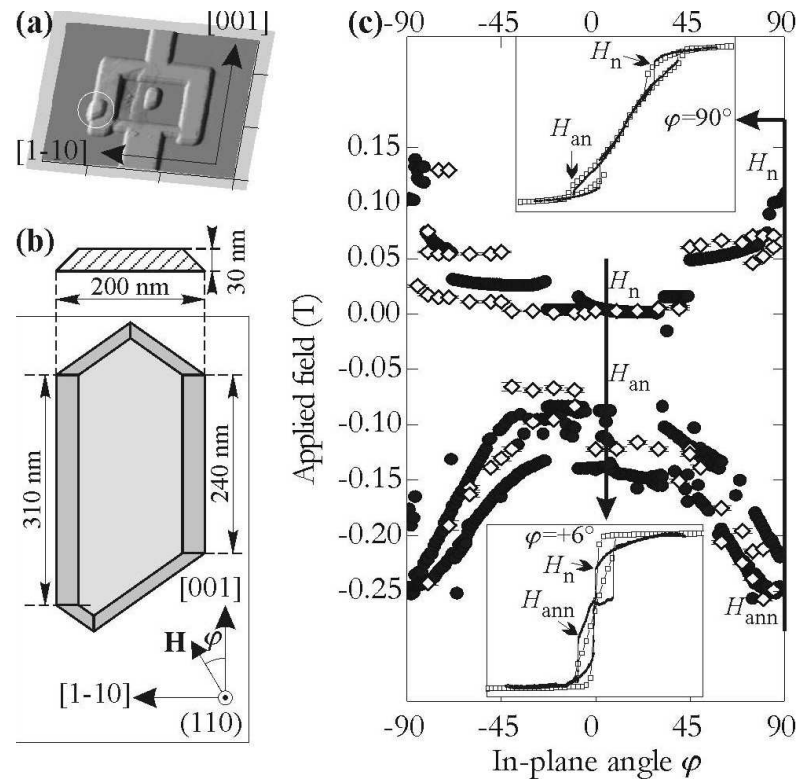

FIGURE 3. (a) AFM picture of the micro-SQUID. The dot strongly coupled to the micro-SQUID is indicated by a circle. (b) top view of the dot (c) Plot of $H_{\text {nuc }}$ (positive) and $H_{\text {ann }}$ (negative) for experiments (full symbols) and simulations (open symbols). Insets : experimental and simulated loops for two angles.

\section{REFERENCES}

[1] P. Jubert, O. Fruchart, C. Meyer, Self-assembled growth of facetted epitaxial fe(110) islands on mo(110), Phys. Rev. B 64 (2001) 115419.

[2] P. O. Jubert, J. C. Toussaint, O. Fruchart, C. Meyer, Y. Samson, Flux-closure-domain states and demagnetizing energy determination in sub-micron size magnetic dots, Europhys. Lett. 63 (1) (2003) 135-141.

[3] J. C. Toussaint, A. Marty, N. Vukadinovic, J. Ben Youssef, M. Labrune, A new technique for ferromagnetic resonance calculations, Comput. Mater. Sci. 24 (1-2) (2002) 175-180.

[4] K. Ramstöck, T. Leibl, A. Hubert, Optimizing stray field computations in finite-element micromagnetics, J. Magn. Magn. Mater. 135 (1994) 97.

[5] H. A. M. V. den Berg, A micromagnetic approach to the constitutive equation of soft-ferromagnetic media, J. Magn. Magn. Mater. 44 (1984) 207.

[6] W. Rave, A. Hubert, Magnetic ground state of a thin-film element, IEEE Trans. Magn. 36 (2001) 3886.

[7] A. Hubert, R. Schäfer, Magnetic domains. The analysis of magnetic microstructures, Springer, Berlin, 1999.

[8] W. Wernsdorfer, Classical and quantum magnetization reversal studies in nanometer-sized particles and clusters, in: I. Prigogine, S. A. Rice (Eds.), Advances in Chemical Physics, Vol. 118, Wiley, 2001.

[9] K. Y. Guslienko, K. L. Metlov, Evolution and stability of a magnetic vortex in a small cylindrical ferromagnetic particle under applied field, Phys. Rev. B 63 (2001) 100493(R). 